\title{
A Modification of the In Vitro Canine Purkinje Fiber Repolarization Assay using Variable Frequency Pacing
}

\author{
A Safer ${ }^{1}$, G Gintant ${ }^{2}$ \\ ${ }^{1}$ Abbott GmbH\&Co.KG, R\&D IT Systems, Ludwigshafen, Germany \\ ${ }^{2}$ Abbott Inc., GPRD Integrative Pharmacology, Abbott Park, IL, USA
}

\begin{abstract}
The in vitro canine cardiac Purkinje fiber (CCPF) assay used to predict drug-induced $Q T$ prolongation typically employs trains of constant frequency stimulation. We describe a modification of the model using a variable pacing protocol that mimics the sinus arrhythmia of living dogs.

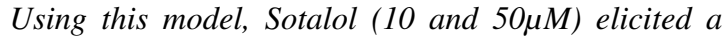
dose-dependent shift of the variable frequency pacing return curve in a dose dependent manner to lower diastolic intervals. The shift and increased variability of the return curve with the high Sotalol concentration represents greater electrophysiologic instability.

Further investigations are needed to assess the utility of the variable pacing protocol to evaluate electrophysiologic instability as a surrogate marker for proarrhythmia in the CCPF model.
\end{abstract}

\section{Introduction}

The ICH safety pharmacology guidance (S7B) [1] on QT interval prolongation by human pharmaceuticals specified in vitro methods for repolarization and conductance abnormalities as preclinical studies to identify adverse pharmacodynamic effects. The canine cardiac Purkinje fiber (CCPF) assay is a frequently used in vitro model, deriving action potential duration (APD) measurements using constant pacing stimulation.

Drug-induced changes in repolarization of action potentials from isolated Purkinje fibers reflect effects of drugs on multiple ionic channels [2]. These changes provide insight to the mechanisms underlying the genesis of arrhythmias. Thus, the Purkinje fiber assay has proven useful in predicting clinical outcomes [11, 12]. However, experimental conditions typically used fail to fully mimic conditions in vivo, including the sinus arrhythmia prominent in some species (including canines). We therefore questioned whether model performance could be improved by applying stimulation protocols resembling normal canine rhythms. Interestingly, only a relatively few prior studies have employed variable pacing with the CCPF model [3-8].

\subsection{Biological background}

The cells of Purkinje fibers are organized into several tracts that lead away from the atrioventricular node and ramify into the mass of ventricular tissue, distributing the electrical signal initiating contraction. From their termini the signal is spread through the working myocardium. Purkinje fibers are not nerves, but are actually modified strands of cardiac muscle that form nerve-like structures. Purkinje fibers are minimally contractile due to a paucity of actin and myosin fibrils. Purkinje fibers do have some of the other features of cardiac myocytes, including a centrally located single nucleus, and even intercalated disks. All muscle tissue is "excitable" and this property can be used to carry information [10].

The canine cardiac rhythm is subject to a much greater variability compared to man due to a higher degree of responsiveness of the heart rate (RR cycle) to respiration [3]. The range of beat-to-beat changes the defining sinus arrhythmia is between 20 and $80 \mathrm{~ms}$, while Beagle dogs operate in a range of 700 to $1000 \mathrm{~ms}$ (see Figure 1).

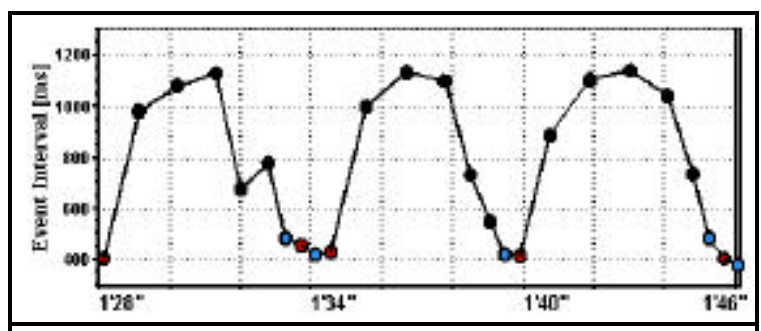

Figure 1. Typical canine heartbeat interval example showing variability over 3 respiration cycles [ms].

The long RR intervals (slow heartbeat) usually do not pose problems to repolarization, while a train of short beat intervals mimicking heart rate acceleration may result in insufficient repolarization (figure 2). Action potentials (APs) arising prior to complete repolarization are termed premature beats. Drug effects on ratedependent alterations of the action potential duration (APD) and refractoriness may be either pro- or antiarrhythmic [6-9, 11]. 


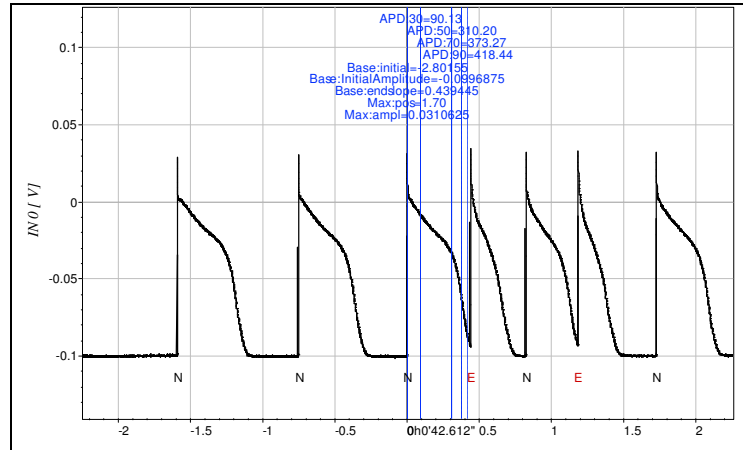

Figure 2. Typical CCPF train of APs using variable pacing frequency.

$\mathrm{N}=$ normal interval AP; $\mathrm{E}=$ premature beat AP

Premature beats are characterized by the lack of return to the resting potential, a reduced rate of depolarization and maximal depolarization amplitude, and a decreased APD (figures 2, 3). If the coupling interval is sufficiently short (short RR interval), the AP shape is significantly altered (as no full depolarization takes place) and sometimes complete block is observed.

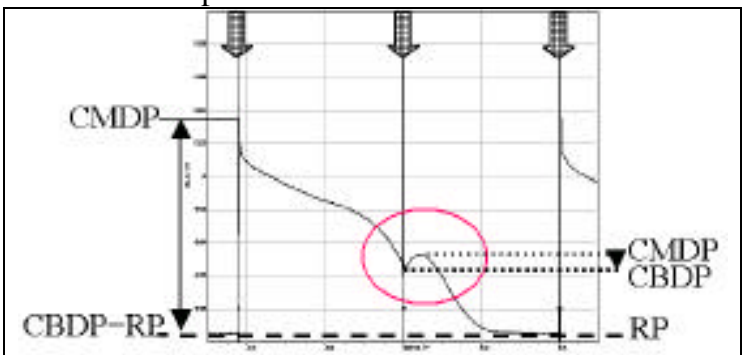

Figure 3. Example of a normal cycle followed by a very early premature beat. $\mathrm{RP}=$ resting potential $\mathrm{CBDP}=$ Cycle basic depolarization level $\mathrm{CMDP}=$ Cycle maximum depolarization level; thin arrows: depolarization gain; gray arrows: position of stimuli.

\subsection{Hypothesis}

The constant frequency stimulation used in the standard CCPF model provides one established measure to evaluate drug effects on repolarization. An alternative approach presented here applies a stimulus protocol obtained from conscious animals, mimicking the strong sinus arrhythmia observed in dogs. The application of variable frequency stimulation in vitro may improve the sensitivity and predictivity of the CCPF model in detecting drug-induced changes of repolarization and proarrhythmic drug activity.

\section{Materials and methods}

We investigated the effect of Sotalol (ascending concentrations of 10 and $50 \mu \mathrm{M}$ ) on repolarization of cardiac Purkinje fibers in vitro using a) constant pacing rates (2000, 800 and $400 \mathrm{~ms}$ basic cycle lengths) and b) variable frequency pacing, with stimulation intervals obtained from telemetry-equipped Beagle dogs.

\subsection{Materials}

Protocols utilized to evaluate drug effects on the Purkinje fiber action potential duration have been described previously [12]. Briefly, larger, free-running canine cardiac Purkinje fibers were excised, placed in warmed (36.5-37.0 ${ }^{\circ} \mathrm{C}$ ) chamber, and superfused (8-10 $\mathrm{mLs} / \mathrm{min}$ ) with preheated Tyrode's solution containing (in $\mathrm{mM}$ ): $\mathrm{NaCl}, 131 ; \mathrm{NaHCO} 3,18 ; \mathrm{NaH} 2 \mathrm{PO} 4,1.8$; $\mathrm{MgCl} 2,0.5$; dextrose, 5.5; $\mathrm{KCl}, 4 ; \mathrm{CaCl} 2,2$ (aerated with $95 \% \mathrm{O} 2 / 5 \% \mathrm{CO} 2$ [pH=7.2 at room temperature]). Fibers were field stimulated $(2 \mathrm{x}$ threshold, biphasic waveform, typically $1-2 \mathrm{msec}$ in duration) using platinum electrodes located in the chamber floor and impaled with $3 \mathrm{M} \mathrm{KCl-filled} \mathrm{microelectrodes} \mathrm{(resistance}$ 10-30 M-ohm); electrical activity was monitored.

\subsection{Equipment}

Transmembrane potentials were obtained using high input impedance electrometers (IE-210, Warner Instruments). Signals were recorded digitally (typically 2 $\mathrm{kHz}, 16$ bit resolution) using a Digidata 1200 hardware and Clamp 8 software (Axon Instruments) and analyzed using the proprietary ABBIOS software program [13]. Statistical calculations were performed on a personal computer using $\mathrm{S}+$ (Insightful Inc.) and RS/1 (BBN Inc.).

\subsection{Methods}

Studies were initiated after a minimum 30-minute equilibration period with stimulation. Fibers were considered suitable for study if (during stimulation at 2 sec basic cycle length) the following criteria were satisfied: a), the membrane potential just prior to action potential upstroke was more negative than $-80 \mathrm{mV}, \mathrm{b}$ ), the APD ranged between 300-500 $\mathrm{msec}$ (spanning approx. 1.2 standard deviations from the mean value of $405 \mathrm{msec}$ ), and c), the normal automatic rate did not exceed the $2 \mathrm{sec}$ stimulation cycle length.

The depolarization gain (DG) was measured as difference between the peak of the action potential upstroke, named cycle maximum depolarization level (CMDP) and the resting potential (RP). The action potential duration to $\mathrm{x} \%$ of DG (APDx) is defined as the time when the repolarization transects the $\mathrm{DG}^{*}(100-\mathrm{x})$ level.

We observed that standard programs use a definition of $\mathrm{DG}=(\mathrm{CMDP}-\mathrm{CBDP})^{*}(100-\mathrm{x}), \mathrm{CBDP}$ being the cycle basic depolarization level. This results in incorrect APDx estimates, with APDx falling too short.

Repolarization duration was measured at 30\%, 50\%, $70 \%, 90 \%$ and $95 \%$ of DG. We report only the usual 
95\% repolarization (APD95) in this work. In cases where the APD95 could not be measured due to prematurity, missing observations occurred.

The DI was calculated as the time from APD95 to the subsequent stimulated upstroke [8]. The return curve (RC) (sometimes named restitution curves in literature) is defined as the relationship between DI of the preceding stimulation cycle and the subsequent APD95.

RCs from variable frequency stimulation (obtained by plotting the diastolic interval vs. the APDx values of subsequent beats interval) were well fitted by:

$$
A P D x=\exp \left\{a+\frac{b}{\left\{1+\exp \left[\left(\frac{c-\log D I}{d}\right)\right]\right\}}\right\} \text {. }
$$

Furthermore, loess-smoothing method was applied (weighting window $20 \%$ or $50 \%$ of observations) as a non-parametric method, and for control of fit by the logistic function. The first derivative of the fitted function, respectively consecutive differences of the loess-smoothed RC were used as an estimate of the slope, and the steepest slope is reported here. The slope of the RC may provide an indication of potential arrhythmogenicity.

A reasonable function estimate of return curves under constant BCL is not very reliable. Thus, we used the last $10 \mathrm{BCL}$ intervals at each BCL level for function fitting to ensure stable APD95 levels. The fitted function was:

$$
A P D 95=\exp \{\text { const }+b * \log (D I-\operatorname{lag})\}
$$

A test with control treatment under constant pacing conditions at 2000, 800 and $400 \mathrm{msec}$ basic cycle length (BCL) was compared to variable frequency stimulation (Table 1) in 3 dog CCPF experiments (E1, E2, E4).

Table 1. Type and number of experiments conducted

\begin{tabular}{lll}
\hline Treatment & Pacing method & $\mathrm{N}$ \\
\hline Control $(\mathrm{CON})$ & Constant & 2 \\
Control & Variable & 3 \\
Sotalol $10 \mu \mathrm{M}$ (SOT10) & Constant & 1 \\
Sotalol $10 \mu \mathrm{M}$ & Variable & 3 \\
Sotalol $50 \mu \mathrm{M}$ (SOT50) & Constant & 2 \\
Sotalol $50 \mu \mathrm{M}$ & Variable & 3 \\
\hline
\end{tabular}

Three more experiments with other compounds (E3, E5 and E6) will be reported in another paper.

\section{Results}

For brevity we restrict our report to only the details of one experiment (E4). Due to protocols, constant and variable frequency pacing span different ranges of BCL (400 to $2000 \mathrm{~ms}$ vs. $345-1275 \mathrm{~ms}$ ). Though there are principally similar results in all three Sotalol experiments, we observed considerable interindividual differences in response on APD/DI slopes.

\subsection{Constant frequency pacing}

Under control conditions, step changes in pacing frequency (from 2000 to $800 \mathrm{msec}$ and from 800 to 400 msec basic cycle length) elicited an exponential adoption function of APD90 that required greater than 1 minute for stabilization to a constant level (Figure 4).

In one of four fibers we observed chaotic response after suddenly shortening the BCL from 800 to $400 \mathrm{~ms}$ during exposure to $50 \mu \mathrm{M}$ Sotalol (Figure 4). The same preparation showed chaotic response to the dynamic pacing protocol.

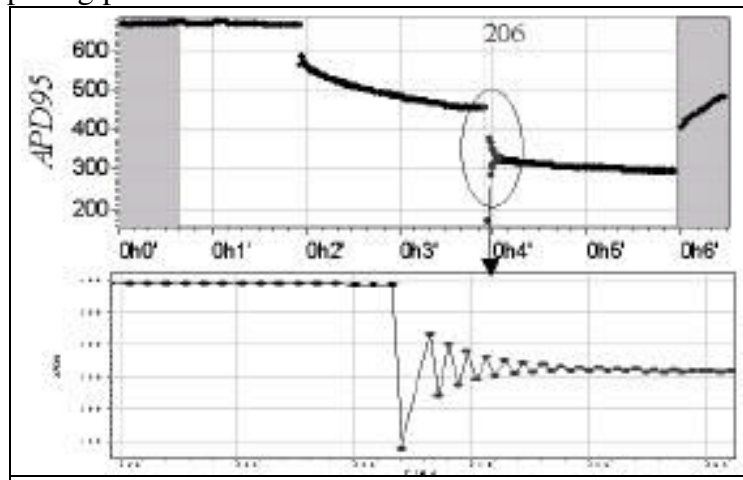

Figure 4. Bifurcation transition (APD alternans) after suddenly BCL shortening (800 to $400 \mathrm{~ms}$ ) in fixed pacing under treatment with Sotalol $50 \mu \mathrm{M}$.

The RC visualization of experiment E4 (Figure 5) shows that return curve slope and maximum asymptote increased dose-dependently, but with considerable interindividual differences.

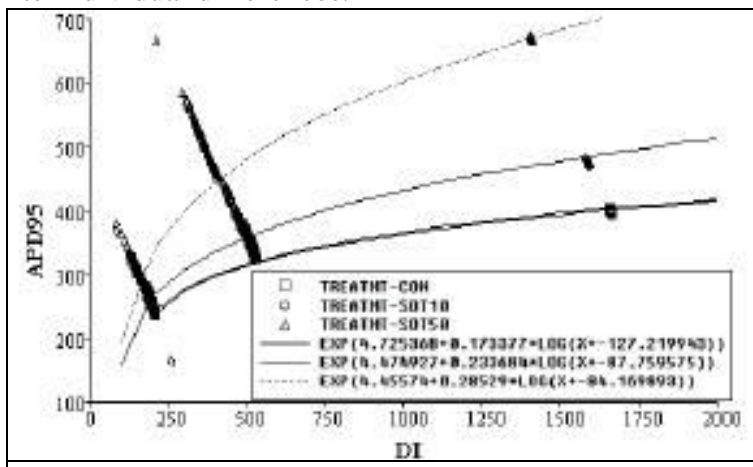

Figure 5. Return curve of E4 under constant pacing. Treatments from bottom to top: CON, SOT10, SOT50.

\subsection{Variable frequency pacing}

An example of a rate transformed return curve of variable frequency pacing is shown in figure 6. A clear separation is seen between the RCs with the slopes and APD95-distances differ considerably. Increased variability is observed in the deviation of stimuli from $\mathrm{RC}$ under Sotalol $50 \mu \mathrm{M}$ compared to Sotalol $10 \mu \mathrm{M}$ and control, which is interpreted as a sign of increasing 
instability and possible pro-arrhythmogenic potential (Figure 6).

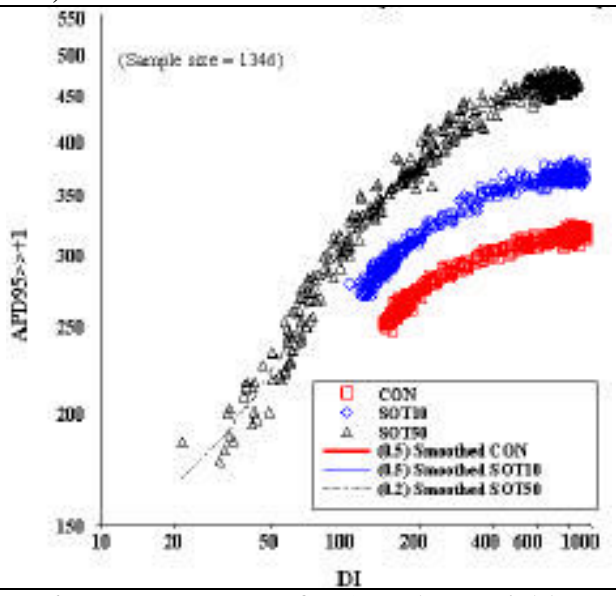

Figure 6. Return curve of E4 under variable pacing Treatments from bottom to top: CON, SOT10, SOT50.

As a summary of experiments we compared the maximum slopes of our experiments with incremental Sotalol concentrations under variable pacing protocol (Figure 7). In two of three experiments, the maximum slope of RC exceeded 1; none of the slopes exceeded 0.8 using the constant pacing protocol. Slopes exceeding 1 have been linked to arrhythmogenicity.

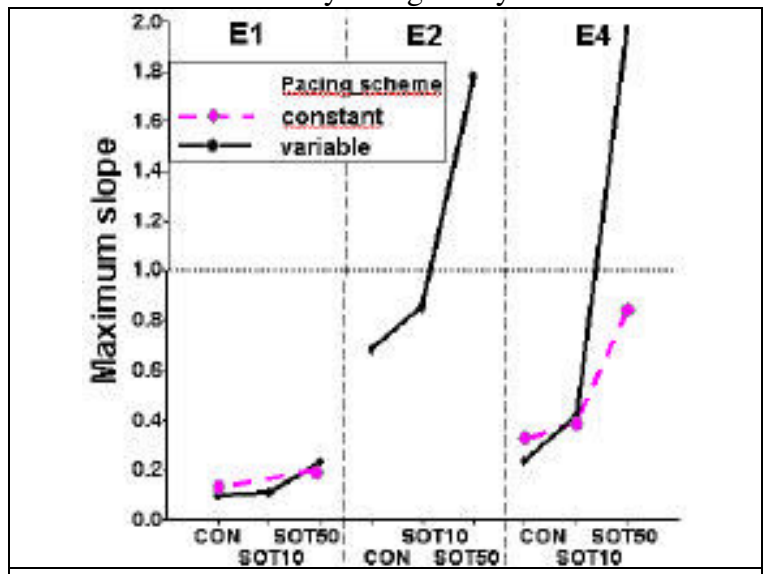

Figure 7. Maximum slope of return curves.

\section{Discussion and Conclusions}

Sotalol elicited a dose-dependent shift of the variable frequency pacing return curve in a dose dependent manner towards increased APDs and lower DI. $50 \mu \mathrm{M}$ Sotalol significantly decreased the diastolic interval. The increased slopes of the return curve with the high Sotalol concentration represent increased electrophysiologic instability.

Further investigations are necessary to assess the utility of the variable pacing protocol and electrophysiologic instability in vitro as a surrogate marker for proarrhythmia in the CCPF model.

\section{Acknowledgements}

Thanks are owed to Jason Palmatier for assisting in the experiments and Sven Mensing who assisted in data handling and creation of an automation tool.

\section{References}

[1] International Conference on Harmonization (ICH). Safety Pharmacology Studies for Assessing the Potential for Delayed Ventricular Repolarization (QT Interval Prolongation) by Human Pharmaceuticals S7B. Draft Consensus Guideline, released Feb. 7, 2002.

[2] Picard S, Lacroix P. QT interval prolongation and cardiac risk assessment for novel drugs. Curr Opin Investig Drugs. 2003;4:303-8.

[3] Colatsky TJ, Hogan PM. Effects of external calcium, calcium channel blocking agents and stimulation frequency on cycle length-dependent changes in cardiac action potential duration. Circ Res 1980;46:543-552.

[4] Elharrar V, Suravic B. Cycle length effects on return of action potential duration in dog cardiac fibers. Am $\mathrm{J}$ Physiol 1983;244:H782-H792.

[5] Elharrar V, Atarashi H, Suravic B. Cycle length dependent action potential duration in canine cardiac Purkinje fibers. Am J Physiol 1984;247:H936-H945.

[6] Chialvo DR, Michaels DC, Jalife J. Supernormal excitability as a mechanism of chaotic dynamics of activation in Purkinje fibers. Circ Res 1990;66:525-545.

[7] Gilmour RF, Otani NF, Watanabe MA. Memory and complex dynamics in cardiac Purkinje fibers. Am J Physiol 1997;272:H1826-H1832.

[8] Koller ML, Riccio ML, Gilmour RF. Dynamic restitution of action potential duration during electric alternans and ventricular fibrillation. Am J Physiol 1998:H1826-H1832.

[9] Watanabe M, Gilmour RF. Biphasic' restitution of action potential duration and complex dynamics in ventricular myocardium. Circ Res 1995;76:915-921.

[10] Caceci T. VM8054 Veterinary Histology. Purkinje Fibers. http://education.vetmed.vt.edu/Curriculum/VM8054/Labs/ Lab12a/EXAMPLES/Exprkfib.htm

[11] Zipes DP. Mechanisms of Clinical Arrhythmias. J Cardiovasc Electrophysiol 2003;14:902-912.

[12] Gintant GA, Limberis JT, McDermott JS, Wegner CD, Cox BF. The Canine Purkinje Fiber: an In Vitro Model System for Acquired Long QT Syndrome and Druginduced Arrhythmogenesis. J Cardiovasc Pharmacol 2001;37:607-618.

[13] Safer A, Bystricky W, Schweizer M. ABBIOS (ABott BIOsignal System): A Workbench System Software for Data Mining in Biosignals, with Applications in Drug Research and Development. Computers in Cardiology 2002;29:649-652.

Address for correspondence.

Anton Safer

Abbott GmbH\&Co.KG

GGOIB (R\&D IT Systems Biometry\&Biosignals)

Knollstrasse 50

D-67061 Ludwigshafen/Rhein, Germany

Email: Anton.Safer@Abbott.Com 